\title{
Postoperative complications and its relationship with the severity of postoperative pain in patients undergoing thoracic surgery
}

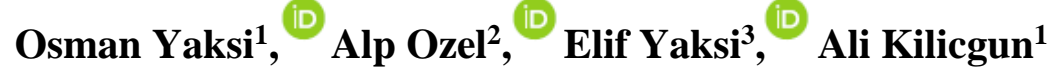 \\ ${ }^{1}$ Department of Thoracic Surgery, Bolu Abant Izzet Baysal University, Faculty of Medicine, Bolu, Turkey \\ ${ }^{2}$ Department of Physiotherapy and Rehabilitation, Bolu Abant Izzet Baysal University, Faculty of Health Sciences, Bolu, \\ Turkey \\ ${ }^{3}$ Department of Physical Medicine and Rehabilitation, Bolu Abant Izzet Baysal University, Faculty of Medicine, Bolu, \\ Turkey
}

\section{ABSTRACT}

Aim: To investigate the complications that occur in our patients who underwent thoracic surgery, as well as the relationship between postoperative pain and complications.

Method: Of the 117 patients who underwent surgery between January 2018 and December 2018, there were 99 patients with pain and the other parameters whose data's were complete. Medical records of the patients were investigated in terms of age, gender, smoking status and frequency, diagnosis, treatment, length of stay in the hospital, postoperative complications and visual analog scale (VAS). The postoperative complications and VAS values were compared statistically.

Results: The mean age of the patients was $50.52 \pm 18.46$ years, $26(26.3 \%)$ patients were female and $73(73.7 \%)$ were male. The average length of stay in hospital was 4.08 \pm 3.06 days and average pain severity was 3.92 \pm 2.07 . The most common diagnosis in our cases was lung cancer, and the most common complication was prolonged air leakage. There was a significant relationship between the severity of pain and the presence of postoperative complications in our patients $(p=0.001)$. However, the correlation relationship was found to be low $(r=0.322)$. Conclusion: The results of our study revealed that optimal postoperative pain control is an important factor for preventing postoperative complications.

Keywords: Thoracic surgery, chest surgery, postoperative complications, pain, visual analog scale.

$\triangle$ Dr. Osman Yakşi

Department of Thoracic Surgery, Bolu Abant Izzet

Baysal University, Faculty of Medicine, Bolu, Turkey.

E-mail: oyaksi@hotmail.com

Received: 2020-11-26 Revised: 2020-12-18

Accepted: 2020-12-29 / Published online: 2021-04-01

\section{Introduction}

Since thoracic surgery is one of the most painful surgical procedures, the severity and duration of the pain associated with this surgery is remarkable. The most common causes of pain after thoracic surgery include multilayer intercostal incisions, rib injury or resection, surgical drains and thoracostomy tube insertion, and suturing technique [1,2]. Acute, moderate to severe pain levels due to this diversity of insults may not significantly decrease during hospital stay or the first month after surgery. In addition, poor management of acute postoperative pain can lead to the development of chronic pain syndromes. Chronic pain can last for years, and even lower pain levels can reduce patient satisfaction [3]. Post-thoracotomy pain syndrome is common in patients undergoing thoracotomy and occurs in approximately $50 \%$ of patients. It is estimated 
that these patients have persistent pain at 6 months and even $20 \%$ may continue to experience pain in 6 to 7 years [4-6]. In a previous study, it was reported that patients described pain one year after thoracotomy and that most continued to report pain even after years [7].

Despite improvements in anesthesia and surgical techniques, the rate of postoperative complications in thoracic procedures is still high, at $27 \%$ [8]. Thus, pulmonary and cardiovascular complications after thoracic surgery result in significant morbidity and mortality, and are also the leading cause of prolonged hospital stay with increased overall cost $[9,10]$. Further, complications compromise return to baseline function, may affect further cancer treatment, and overall quality of life [11]. In addition, there are common patientspecific risk factors such as age, pre-operative adverse pulmonary function tests, cardiovascular comorbidity, smoking status, and chronic obstructive pulmonary disease [12]. The main purpose of this study is to investigate the complications that occur in our patients who underwent thoracic surgery, as well as the relationship between postoperative pain and complications.

\section{Materials and Methods}

We retrospectively evaluated patients who underwent classic postero-lateral thoracotomy in our institution (Bolu Abant Izzet Baysal University, Bolu, Turkey). The study protocol was approved by the ethics committee of Bolu Abant Izzet Baysal University Human Research Ethics Committee (2018/298). Of the 117 patients who underwent surgery between January 2018 and December 2018, there were 99 patients with pain and the other parameters whose data's were complete. Medical records of the patients were investigated in terms of age, gender, smoking status and frequency, diagnosis, treatment, length of stay in the hospital, postoperative complications and visual analog scale (VAS). The postoperative complications and VAS values were compared statistically.

In our clinic, a visual analog scale is used to evaluate the pain intensity of the patients on the first postoperative day. The visual analog scale (VAS) is widely used as an outcome measure, as in this study. It is usually presented as a 100$\mathrm{mm}$ horizontal line on which the patient's pain intensity is represented by a point between the extremes of "no pain at all" and "worst pain imaginable." Its simplicity, reliability, and validity, as well as its ratio scale properties, make the VAS the optimal tool for describing pain severity or intensity $[13,14]$.

\section{Statistical analysis}

Data analysis was performed on SPSS version 20.0 software (SPSS Inc., Chicago, USA). Quantitative parametric data were expressed as mean plus standard deviation (SD), and quantitative non-parametric data as median values with minimum and maximum. The Kolmogorov Smirnov and Shapiro-Wilk test was used to analyze the distribution of variables. For parametric data, correlation between groups were performed using the Pearson correlation analysis. The $p$-value $<0.05$ was significantly considered.

\section{Results}

Of the 117 patients who underwent surgery between January 2018 and December 2018, there were 99 patients with complete data regarding pain and the other parameters. The mean age of the patients was $50.52 \pm 18.46$ years, $26(26.3 \%)$ patients were female and 73 $(73.7 \%)$ were male. The average length of stay in hospital was $4.08 \pm 3.06$ days and the average 
Table 1. Demographic data of the patients

\begin{tabular}{|l|c|}
\hline Parameters & Cases (n=99) \\
\hline $\begin{array}{l}\text { Age (years) } \\
\text { (Mean } \pm \text { SD) }\end{array}$ & $50.52 \pm 18.46$ \\
\hline $\begin{array}{l}\text { Gender, N (\%) } \\
\text { Woman } \\
\text { Male }\end{array}$ & $73(73.7)$ \\
\hline $\begin{array}{l}\text { Duration of stay (days) } \\
\text { (Mean } \pm \text { SD) }\end{array}$ & $4.08 \pm 3.06$ \\
\hline $\begin{array}{l}\text { Cigarettes (pack-years) } \\
\text { (Mean } \pm \text { SD) }\end{array}$ & $18.84 \pm 22.55$ \\
\hline $\begin{array}{l}\text { Smoking status, N (\%) } \\
\text { Yes }\end{array}$ & $59(59.6)$ \\
No & $40(40.4)$ \\
\hline Diagnosis, N (\%) & \\
Nodular lesion in the lung & $20(20.2)$ \\
Lung cancer & $25(25.2)$ \\
Mediastinal LAP & $6(6.0)$ \\
Pneumothorax & $15(15.1)$ \\
Pleural effusion & $9(9.0)$ \\
Pulmonary fibrosis & $5(5.0)$ \\
Pleurisy & $9(9.0)$ \\
\hline $\begin{array}{l}\text { Pain severity (VAS) } \\
\text { (Mean } \pm \text { SD) }\end{array}$ & $3.92 \pm 2.07$ \\
\hline $\begin{array}{l}\text { Postoperative } \\
\text { complications, N (\%) } \\
\text { Yes } \\
\text { No }\end{array}$ & $22(22.2)$ \\
\hline & $77(77.8)$ \\
\hline
\end{tabular}

pain severity on the visual analog scale (VAS) was $3.92 \pm 2.07$. Fifty-nine $(59.6 \%)$ patients were tobacco smokers (18.84 \pm 22.55 packyears). The demographic data of the individuals included in the study are shown in Table 1 . The five most common diagnoses in our patients were as follows: lung cancer $(n=25,25.25 \%)$, nodular lesions in the lung $(n=20,20.20 \%)$, spontaneous pneumothorax $(\mathrm{n}=15,15.15 \%)$, pleural effusion $(\mathrm{n}=9, \quad 9.09 \%)$, and hyperhidrosis $(\mathrm{n}=8,8.08 \%)$ (Table 1). Two patients were revised (air leakage $n=1$, hemorrhage $n=1$ ).
Mortality occurred in one patient after surgery (1.01\%).

Five of the most common complications we encountered in our cases were prolonged air leakage $(n=4,14.8 \%)$, atrial fibrillation $(n=3$, $11.1 \%)$, hematoma $(\mathrm{n}=2,7.4 \%)$, hemorrhage $(n=2,7.4 \%)$, and tachycardia $(n=2,7.4 \%)$. The postoperative complications of the patients are shown in Table 2. There was a significant relationship between the severity of pain and the presence of postoperative complications in our patients $(p=0.001)$ (Table 3$)$.

Table 2. Postoperative complications.

\begin{tabular}{|l|c|}
\hline $\begin{array}{l}\text { Postoperative } \\
\text { complication }\end{array}$ & $\mathbf{N}(\boldsymbol{\%})$ \\
\hline Atrial Fibrillation & $3(11.1)$ \\
\hline Empyema & $1(3,7)$ \\
\hline Apical bull perforation & $1(3,7)$ \\
\hline Arintenoid dislocation & $1(3,7)$ \\
\hline Atelectasis & $1(3,7)$ \\
\hline Delirium & $1(3,7)$ \\
\hline Diaphragm elevation & $1(3,7)$ \\
\hline Dyspnea & $1(3,7)$ \\
\hline Embolism & $1(3,7)$ \\
\hline Hematoma & $2(7.4)$ \\
\hline Hemorrhage & $2(7.4)$ \\
\hline Hypertension & $1(3,7)$ \\
\hline Constipation & $1(3,7)$ \\
\hline Pericardial effusion & $1(3,7)$ \\
\hline Secretion retention & $1(3,7)$ \\
\hline Hoarseness & $1(3,7)$ \\
\hline Chylothorax & $2(7.4)$ \\
\hline Tachycardia & $4(14.8)$ \\
\hline Prolonged air leakage & \\
\hline
\end{tabular}




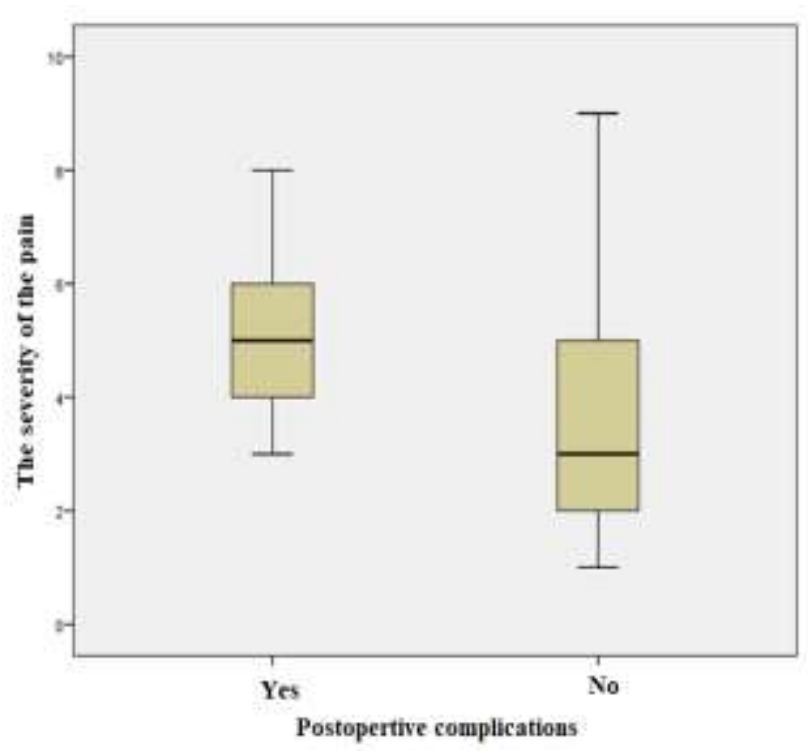

Figure 1. The relationship between the severity of pain and the presence of postoperative complications.

\section{Discussion}

In our study, a weak relationship was found between the severity of pain and the presence of postoperative complications. Therefore, at least $10 \%(\mathrm{r} 2)$ of the pain intensity in the correlation analysis could explain the postoperative complications. In addition, the rate of postoperative complications in the patients included in our study was found to be compatible with the literature [15]. Further, as in our study, Boffa et al. reported an average of 5 days of hospital stay in their extensive study [16].

Thoracic surgery operations are usually performed with one of the classical thoracotomy, minithoracotomy, video-assisted thoracoscopic surgery (VATS) and the latest robot-assisted thoracoscopic surgery (RATS) techniques [1,2,17]. Although classical thoracotomy with a posterolateral incision provides optimal surgical access, it is also a very painful procedure as it involves the division of the latissimus dorsi, serratus anterior, rhomboids, and trapezius muscles. Therefore, thoracotomy intervention requires good management of both postoperative complications and pain control in thoracic surgery patients. In addition, this painful procedure has been found to be an important risk factor for the development of permanent opioid use [1,2,17].

Pulmonary complications are the most important group affecting morbidity, mortality and long-term hospital stay after thoracic surgery, with an incidence of 30-50\% [8]. Complications reported from lungs are prolonged air leak, pneumonia, pulmonary embolism, major atelectasis, adult respiratory distress syndrome, prolonged ventilation and need for tracheostomy [18]. In our study, the most common postoperative complications were prolonged air leakage, atrial fibrillation, tachycardia, hematoma, and hemorrhage. Impaired pulmonary function was the most studied risk factor for lung resection because it is reasonable to assume that a surgeon is the most important factor to consider when weighing the risk of lung resection [19].

Since thoracic surgery operations are one of the most painful surgical procedures, good pain management is crucial for postoperative comfort and increased mortality and morbidity [20]. As we routinely practice in our patients, opioids and NSAIDs are often sufficient to provide optimal pain control in patients undergoing VATS and sternotomy [20]. In addition, performing minimal surgical procedures, epidural analgesia, intercostal block are applications that may be required to provide good analgesia. In a study by Landreneau $\mathbf{J}$ et al [21], VATS was applied to 81 patients and thoracotomy to 57 patients. Patients who underwent VATS experienced significantly less postoperative pain and required less post-surgery narcotic analgesia. On the other hand, elderly patients are at a higher risk of postoperative complications, and 
the severity of pain in these patients may further increase the complication rate. Therefore, analgesic methods to reduce postoperative complications in this group of patients should be chosen more carefully. In a review about the use of regional analgesia, it was reported that these methods reduce perioperative morbidity, but in addition to the side effects of the drugs used, complications such as epidural hematoma and infection may develop [22]. In our patients, there was a significant relationship between the severity of pain and the presence of postoperative complications. However, the correlation relationship was found to be low.

Consequently, for patients scheduled for thoracic surgery, a successful postoperative outcome can be achieved if several conditions are met. These include optimizing patientrelated risk factors such as gender, extremely low or extremely high body mass index, nicotine consumption history or pulmonary comorbidities when surgery is indicated, pulmonologist and postoperative intensive care support, choosing the right incision approach, early physical therapy. In addition, the results of our study revealed that optimal postoperative pain control is an important factor in preventing postoperative complications. However, the fact that our study is retrospective is as a limiting factor. Therefore, we believe that more comprehensive new studies should be carried out on this subject.

Funding: There is no financial support and sponsorship.

Conflict of Interest: The authors declare that they have no conflict of interest.

Ethical statement: The study protocol was approved by the ethics committee of Bolu Abant Izzet Baysal University Human Research Ethics Committee (2018/298).

\section{Open Access Statement}

This is an open access journal which means that all content is freely available without charge to the user or his/her institution under the terms of the Creative Commons Attribution NonCommercial License (http://creativecommons.org/licenses/bync/4.0). Users are allowed to read, download, copy, distribute, print, search, or link to the full texts of the articles, without asking prior permission from the publisher or the author.

\section{References}

[1]Marshall K, McLaughlin K. Pain Management in Thoracic Surgery. Thorac Surg Clin. 2020;30(3):339-46.

[2]Elmore B, Nguyen V, Blank R, et al. Pain Management Following Thoracic Surgery. Thorac Surg Clin. 2015;25(4):393-409.

[3]Gottschalk A, Cohen SP, Yang S, et al. Preventing and treating pain after thoracic surgery. Anesthesiology: The Journal of the American Society of Anesthesiologists. 2006;104(3):594-600.

[4]Kolettas A, Lazaridis G, Baka S, et al Postoperative pain management. J Thorac Dis. 2015;7(Suppl 1):S62-72.

[5]Bayman EO, Brennan TJ. Incidence and severity of chronic pain at 3 and 6 months after thoracotomy: meta-analysis. J Pain. 2014;15(9):887-97.

[6]Karmakar MK, Ho AM. Postthoracotomy pain syndrome. Thoracic surgery clinics. 2004;14(3):345-52.

[7]Mongardon N, Pinton-Gonnet C, Szekely B, et al. Assessment of chronic pain after thoracotomy: a 1-year prevalence study. The Clin J Pain. 2011;27(8):677-81.

[8]Fernandez FG, Kosinski AS, Furnary AP, et al. Differential effects of operative complications on survival after surgery for 
primary lung cancer. J Thorac Cardiovasc Surg. 2018;155(3):1254-1264.e1.

[9]Kaufmann K, Heinrich S. Minimizing postoperative pulmonary complications in thoracic surgery patients. Curr Opin Anaesthesiol. 2021;34(1):13-19.

[10] Keshava HB, Boffa DJ. Cardiovascular Complications Following Thoracic Surgery. Thorac Surg Clin. 2015;25(4):371-92.

[11] Pedoto A, Perrino AC Jr. Delayed recovery following thoracic surgery: persistent issues and potential interventions. Curr Opin Anaesthesiol. 2019;32(1):3-9.

[12]Licker MJ, Widikker I, Robert J, et al. Operative mortality and respiratory complications after lung resection for cancer: impact of chronic obstructive pulmonary disease and time trends. The Annals of thoracic surgery. 2006;81(5):1830-37.

[13]Bodian CA, Freedman G, Hossain S, Eisenkraft JB, Beilin Y. The visual analog scale for pain: clinical significance in postoperative patients. Anesthesiology. 2001;95(6):1356-61.

[14] Katz J, Melzack R: Measurement of pain. Surg Clin North Am 1999; 79: 231-52

[15] García-Miguel FJ, Serrano-Aguilar PG, López-Bastida J. Preoperative assessment. Lancet. 2003;362(9397):1749-57.

[16]Boffa DJ, Allen MS, Grab JD, et al. Data from The Society of Thoracic Surgeons General Thoracic Surgery database: the surgical management of primary lung tumors. J Thorac Cardiovasc Surg. 2008;135(2):247-54.

[17] Brescia AA, Harrington CA, Mazurek AA, et al. Factors Associated With New Persistent Opioid Usage After Lung Resection. Ann Thorac Surg. 2019;107(2):363-68.
[18] Wright CD, Gaissert HA, Grab JD, et al. Predictors of prolonged length of stay after lobectomy for lung cancer: a Society of Thoracic Surgeons General Thoracic Surgery Database risk-adjustment model. Ann Thorac Surg. 2008;85(6):1857-65; discussion 1865.

[19]Berrisford R, Brunelli A, Rocco G, et al. Audit and guidelines committee of the European Society of Thoracic Surgeons; European Association of Cardiothoracic Surgeons. The European Thoracic Surgery Database project: modelling the risk of inhospital death following lung resection. Eur J Cardiothorac Surg. 2005;28(2):306-11.

[20]Hazelrigg SR, Cetindag IB, Fullerton J. Acute and chronic pain syndromes after thoracic surgery. Surg Clin North Am. 2002;82(4):849-65.

[21]Landreneau RJ, Hazelrigg SR, Mack MJ, et al. Postoperative pain-related morbidity: video-assisted thoracic surgery versus thoracotomy. Ann Thorac Surg. 1993;56(6):1285-89.

[22] Hanna MN, Murphy JD, Kumar K, et al. Postoperative pain management in the elderly undergoing thoracic surgery. Thorac Surg Clin. 2009;19(3):353-61. 